\title{
Deutschland und seine Migranten
}

\section{Vom Werden und vom Selbstverständnis eines Einwanderungslandes}

\author{
THOMAS KUNZ \\ Prof. Dr. Thomas Kunz, Diplom- \\ Politologe, hat eine Professur am \\ Fachbereich Soziale Arbeit und \\ Gesundheit der Frankfurt University \\ of Applied Sciences. Seine Arbeits- \\ schwerpunkte sind: Migration und \\ Soziale Arbeit, Konstruktion und \\ Analyse gesellschaftlicher Fremd- \\ heitsbilder, Sicherheitsdiskurs, \\ Rassismusforschung, Integrations- \\ politik und Integrationssteuerung, \\ Monitoring, Evaluation und Indi- \\ katorenbildung im Kontext von \\ Integration. \\ mtkunz@fb4.fh-frankfurt.de
}

\author{
Die Geschichte der Einwanderung und ihrer \\ Interpretation verlief in Deutschland in mehreren \\ Phasen. Zwischenzeitlich gilt die Beschreibung \\ eines "Einwanderungslandes" als öffentlich \\ anerkannte Status. Doch neue Bezeichnungen \\ lassen alte Vorurteile nicht verschwinden.
}

Der nachfolgende Beitrag beabsichtigt, neben einer Heranführung an das Thema Migration in Deutschland, die Frage zu diskutieren, welches gesellschaftliche Selbstverständnis dem sogenannten Einwanderungsland Deutschland aktuell angemessen scheint. Nachdem die Bundesrepublik im öffentlichen Diskurs seit längerem als Einwanderungsland tituliert wird, erscheint es fraglich, ob bereits ein Selbstverständnis, welches diesem Sachverhalt gerecht wird, existiert.

Die fragwürdige und mit rassistischen Ideologemen unterlegte Behauptung Sarrazins, nach der Deutschland sich abschaffe, lässt sich in diesem Kontext heranziehen und als Abwehrversuch deuten, der gegen solch ein neues Selbstverständnisses gerichtet ist, welches angesichts der demographischen Entwicklung längst überfällig scheint und sich in gesellschaftspolitischen Debatten zumindest ankündigt.

Jenes neue Selbstverständnis ließe auch Ansätze und Konzepte Sozialer Arbeit in der Migrationsgesellschaft nicht unberührt, die - immer noch - nicht unwesentlich auf einer Sonderbetrachtung eines Bevölkerungsteils mit sogenanntem Migrationshintergrund aufruhen, der von einem Bevölkerungsteil ohne Migrationshintergrund unterscheidbar wäre.

\section{Von Menschen mit Migrationshintergrund}

Die Kategorie "Migrationshintergrund « bedarf einer grundsätzlichen Vorbemerkung. Personen mit Migrationshinter- grund sind - auch wenn das Thema gesellschaftlich evident zu sein scheint keine selbstverständlicher, dem Akt des Beobachtens vorgängiger Gegenstand. Vielmehr sind bereits in die scheinbar bloße Feststellung ihrer Existenz umfänglich gesellschaftliche Beobachtungsroutinen, Differenzsetzungen und Konstruktionsprozesse eingeschrieben.

Im Zuwanderungsdiskurs, der auch als Migrations- oder Integrationsdiskurs paraphrasiert werden kann, wird mit diesem Begriff überwiegend eine Differenzlinie gültig gemacht, nach der Gesellschaftsmitglieder entlang der Frage unterschieden werden, ob sie (oder ihre Angehörigen) zugewandert seien oder nicht. Ein Migrationshintergrund gilt als Synonym für ethnische Andersheit, welche mit Zuwanderung in Verbindung stehe und konstruiert ein Sie-Gruppe.

Von den als Zuwanderern oder als Nachkommen von Zugwanderten Identifizierten grenzt sich implizit der Bevölkerungsteil ab, dessen Angehörige sich selbst als Nichtzuwanderer sehen oder angesehen werden. Damit konstituiert sich zugleich eine soziale Gruppe im Sinne einer Wir-Gruppe, die ihre Gruppenzugehörigkeit national begründet (»wir Deutschen «). Aus der Wir-Gruppenposition heraus werden bestehende, mit den WirSie-Gruppenzuordnungen korrelierende unterschiedliche soziale Stellungen und Ansprüche legitimiert und es werden besondere Erwartungen gegenüber $\mathrm{Zu}$ gewanderten abgeleitet. Beispielsweise die, dass wer zu »uns « komme, sich auch anpassen, sich integrieren müsse etc. $\rightarrow$ 
Die Bevölkerungsteil der Menschen mit Migrationshintergrund ist so betrachtet ein Artefakt, ein soziales Konstrukt. Weder existiert er als Gruppe an sich, noch konstituiert er sich als solcher, dennoch ist er Teil gesellschaftlicher Realität. Erst durch soziale und individuelle Praxen, die auf diese Kategorien zurückgreifen und gesellschaftliche Kommunikationen durchziehen, werden diese wirkmächtig, etablieren sich also als subjektive gesellschaftliche Realitäten.

\section{Einwanderungsland, Migrations- oder Zuwanderungsgesellschaft?}

Mit der im Fokus stehenden Dimension ist eine Konzept von Migration verbunden, welches die geographische Verlagerung des Lebensmittelpunktes von Menschen aus nationalstaatlicher Raumperspektive heraus interpretiert. Es ruht wesentlich auf der Binarität von »Herkunftsland/Ankunftsland « (Schröer/Schweppe, 2010, S. 92) auf, macht "nationale Grenzen zur natürlichen Einheit der Forschung « (ebd.) und setzt »Gesellschaft mit Nationalgesellschaft« (ebd.) gleich.

Diese Perspektive mag die hegemoniale sein, zwingend ist sie nicht. Konkurrierende Ansätze, wie beispielsweise die Transmigrationsforschung, eröffnen quer dazu liegende Interpretationsangebote und Realitätsbeschreibungen (vgl. ebd.). Die nachfolgende Betrachtung bezieht sich überwiegend auf jenes etablierte Migrationskonzept, da dieses die herrschenden soziodemographischen Beschreibungsroutinen ebenso durchzieht wie es die historische zuwanderungspolitische Entwicklung prägte und prägt.

\section{Zuwanderungsgeschehen und demographische Entwicklung}

Hinsichtlich des Migrationsgeschehens insgesamt, das heißt hinsichtlich der Zahl der $\mathrm{Zu}$ - und Fortzüge sowohl deutscher wie auch ausländischer Staatsangehöriger über die Grenzen Deutschlands hinweg aufs jeweilige Jahr betrachtet, ließe sich die Frage, ob Deutschland tatsächlich ein Einwanderungsland ist, entsprechend dem jährlichen Wanderungssaldo mit teils wechselndem Ergebnis beantworten.

Demnach war in den Jahren 2008 und 2009 beispielsweise ein (wenn auch nur vergleichsweise niedriger) negativer Wanderungssaldo zu konstatieren, in diesen
Jahren wanderten also mehr Personen ab als zu. (Vgl. BMI, 2014, S. 14 f.) Betrachtet man die Zeitreihe der Jahre 1991 bis 2012 kann festgehalten werden, dass das Gesamtzuwanderungsgeschehen in der Bundesrepublik, also der Saldo der $\mathrm{Zu}$ - und Fortzüge über Jahrzehnte hinweg positiv war. In den Jahren 2010, 2011 und 2012 fiel der Gesamtsaldo im Vergleich zu den vorherigen Jahren wieder höher aus (vgl. ebd., S. 14). »Das Zuwanderungsgeschehen nach Deutschland ist seit Jahren vor allem durch Zuwanderung aus anderen europäischen Ländern bzw. Abwanderung in andere europäische Staaten gekennzeichnet. So kamen im Jahr 2012 fast vier Fünftel aller zuwandernden Personen $(77,5 \%)$ aus einem anderen europäischen Staat nach Deutschland.« (Ebd., S. 16)

Unabhängig davon, ob der Wanderungssaldo positiv oder negativ ausfällt, lässt sich festhalten, dass die Gesellschaft in Deutschland in vielfältiger Weise durch Zuwanderungsprozesse beeinflusst wurde und geprägt wird. Dies betrifft sowohl die Zusammensetzung der Bevölkerung wie auch das gesellschaftliche Selbstverständnis.

Bezeichnungen wie Einwanderungsland oder Migrationsgesellschaft unterstreichen diesen Sachverhalt. Die affirmative wie die pejorative Bezugnahme auf diese Bezeichnungen markieren grundsätzliche, sich gegensätzlich zueinander verhaltende migrationspolitische Positionen.

Ob Deutschland ein Einwanderungsland ist, wurde - wie bereits angedeutet - lange kontrovers diskutiert, wird aber mittlerweile kaum noch bestritten. Strittig ist eher die exakte Terminologie, ob also der Begriff »Einwanderungsland « oder "Zuwanderungsland « die Wahrnehmung der damit in Verbindung gebrachten Migrationsphänomene zur sehr auf Immigration reduziert und andere Migrationstypen (wie beispielsweise sogenannte Trans- und Pendelmigration) ausblendet. Aus kritischer Perspektive wird hier zum Beispiel der Begriff »Migrationsgesellschaft « als terminologisch angemessener favorisiert. (Vgl. Mecheril, 2010, S. 11)

\section{Vom Ausländer- und Ausländerinnenkonzept ...}

Über Jahre hinweg beeinflussten Zuund Fortzüge die demografische Zusammensetzung der Wohnbevölkerung. Als
Indikator hat sich der sogenannte Ausländeranteil etabliert. Differenziert man nach Staatsangehörigkeit, unterscheidet also die Bevölkerungszusammensetzung entlang deutscher oder ausländischer Staatsangehörigkeit, beträgt der Ausländeranteil in der Bundesrepublik aktuell 7,9 Prozent (vgl. BMI, 2014, S. 290 f.).

Darüber hinaus lassen sich je nach Staatsangehörigkeit unterschiedliche große Nationalitätengruppen identifizieren. Auf Basis entsprechender Statistiken zeigt sich, dass Personen mit türkischer Staatsangehörigkeit die größte Gruppe innerhalb der ausländischen Wohnbevölkerung ausmachen, gefolgt von italienischen und polnischen Staatsangehörigen.

Das heißt jedoch nicht, dass türkische Staatsangehörige die größte aus dem Ausland zugewanderte Gruppe darstellen. Diese Betrachtungsweise lässt zum Beispiel die Gruppe der sogenannten (Spät-) Aussiedler außer acht, die zwar zugewandert sind, per definitionem aber als deutsche Staatsangehörige gezählt werden (abgesehen von deren gegebenenfalls ausländischen Familienangehörigen). Laut Statistik sind in der Zeit von 1950 bis 2007 circa 4,5 Millionen Personen (Familienangehörige eingeschlossen) im Rahmen des (Spät) Aussiedlerzuzugs zugereist. Im Jahr 2007 hielten sich davon noch circa 2,8 Millionen (Spät-) Aussiedler in Deutschland auf. (Vgl. BAMF, 2009, S. 19)

\section{... zum Migrationshintergrund}

Nachdem das Zuwanderungsgeschehen die bundesrepublikanische Bevölkerungsentwicklung direkt und zunehmend auch intergenerativ prägt, wurde mit der Einführung der Kategorie »Migrationshintergrund « versucht, dieser Entwicklung auch in der demografischen Beobachtung Rechnung zu tragen. Nicht zuletzt auch aus dem Grund, da mit dem »Ausländer- und Ausländerinnen-Konzept ", also der Unterscheidung nach Staatsangehörigkeit, lediglich ein geringerer Teil der Bevölkerung erfasst werden konnte.

Zur Gruppe der »Personen mit Migrationshintergrund « zählen laut Zensus 2011 »alle zugewanderten und nicht zugewanderten Ausländer/-innen sowie alle nach 1955 auf das heutige Gebiet der Bundesrepublik Deutschland zugewanderten Deutschen und alle Deutschen mit zumindest einem nach 1955 auf 
das heutige Gebiet der Bundesrepublik Deutschland zugewanderten Elternteil « (Statistisches Bundesamt, 2014, S. 38).

Dies ist eine relativ einfache Definition, die den Kerngehalt dieser Kategorie jedoch im Wesentlichen umreißt. Es finden sind in der amtlichen Bevölkerungsstatistik detailliertere und weiter differenzierende Definitionen, die mehr Aufschluss über terminologische Abgrenzungen, aber auch über bestehende Erhebungsprobleme geben (vgl. Statistisches Bundesamt, 2012, S. 364 ff.).

Bezieht man sich auf die erst seit dem Mikrozensus 2005 in der amtlichen Statistik etablierte Kategorie »Migrationshintergrund « stellt sich die Relation anders dar: Laut dem Zensus 2011 verfügen demnach circa 15 Millionen Einwohnerinnen und Einwohner, also etwa ein Fünftel $(18,9 \%)$ der Wohnbevölkerung Deutschlands über einen sogenannten Migrationshintergrund. Hiervon sind wie bereits erwähnt nur rund 6 Millionen ausländischer Staatsangehörigkeit. Den größeren Teil, also circa 9 Millionen Personen, stellen deut- sche Staatsangehörige. (Vgl. Statistisches Bundesamt, 2013, S. 7 u. 10).

Einerseits scheint die Umstellung auf das neue bevölkerungsstatistische Unterscheidungsmerkmal Migrationshintergrund und die Zunahme des damit identifizierten Bevölkerungsanteils die demographische Bedeutung des Migrationsphänomens zu bekräftigen: im Vergleich zum bisherigen Ausländeranteil, der ein knappes Zehntel der Wohnbevölkerung ausmachte, ist der Anteil der Bevölkerung mit Migrationshintergrund nunmehr doppelt so hoch.

\section{Neue Kategorie, alte Entgegensetzung}

Die Einführung dieser neuen Kategorie ist aber auch kritisch zu bewerten: Die Begriffe »Ausländer « und »Migrationshintergrund « werden häufig in der Bevölkerungsstatistik (und auch in anderen Statistiken wie Bildungs- und Arbeitslosenstatistiken) zur Konstruktion von Gruppenbezeichnungen herangezogen, um Migrationsprozesse und Integrati- onsgeschehen zu dokumentieren und zu beobachten. In Folge dessen hat sich die Rede von den Ausländerinnen und Ausländern, den Migrantinnen und Migranten oder den Menschen mit Migrationshintergrund im Zuwanderungsdiskurs fest etabliert.

Die mittels dieser Etiketten zu scheinbar homogenen Bevölkerungsgruppen aggregierten Einzelpersonen unterscheiden sich untereinander jedoch auf vielfältige Weise hinsichtlich Herkunftsländern, Aufenthaltstiteln und Formen der Zuwanderung (zu Zuwanderungsformen und Zuwanderergruppen siehe beispielsweise BMI, 2014, S. 39 ff.; wobei Illegalisierte hier ausgeblendet werden), Aufenthaltsdauer, Lebenslagen sowie anderen demographischen und sozioökonomischen Merkmalen (vgl. beispielsweise Kunz, 2008, 70 f.).

All dies verweist auf die große Heterogenität innerhalb dieses Teils der Bevölkerung. Eine Heterogenität, die sich zum Beispiel auch darin niederschlägt, dass Unterschiede zwischen einzelnen Personen mit Migrationshintergrund,

\section{Die Sicht- und Verfügbarkeit des Migrationshintergrundes im Alltag}

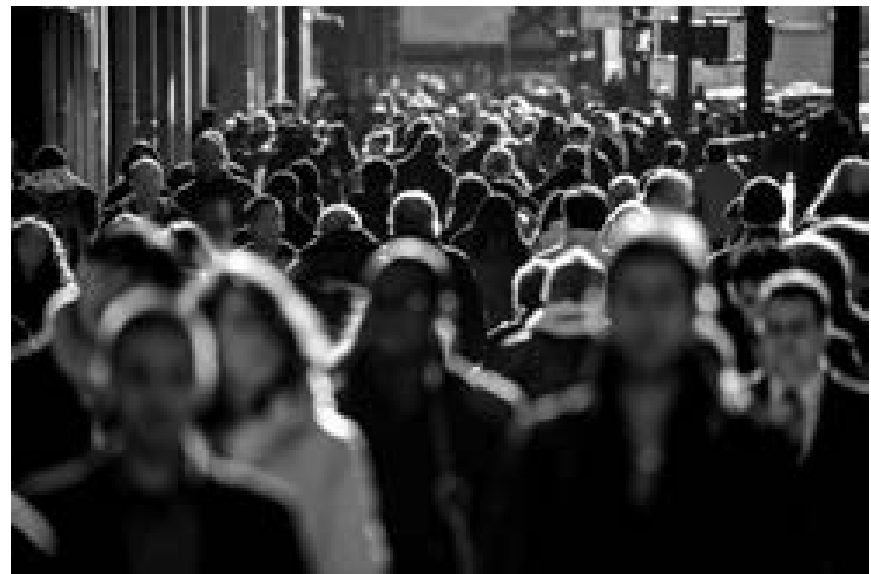

"Woher kommst Du eigentlich?"

Das demographische Abstraktum Migrationshintergrund ist in Alltagskommunikationen zunächst nicht offensichtlich. Die abstrakte Kategorie findet jedoch (wie schon ihre Vorläufer "Gastarbeiter", "Ausländer" etc.) eine Entsprechung in einem hegemonialen Zeichenreservoire, welches herangezogen und gültig gemacht wird, um migrationsbedingte Andersheit zu fixieren und identifizier- und kommunizierbar zu machen. Medien haben hieran einen nicht zu unterschätzenden Anteil. (Vgl. Kunz, 2011)

Es existiert ein Fundus sprachlicher und nichtsprachlicher Zeichen, welche als alltagsweltliche Übersetzung des Migrationshintergrundes gelten: der Rückgriff auf Körpermerkmale (insbesondere Hautfarbe, aber auch Gesichtszüge oder Haartracht), auf Kleidungsstücke und Kleidungsstile, auf das Namensmerkmal, auf Religionszugehörigkeit, aber auch auf Sprach- und Ernäh- rungsgewohnheiten spielt eine exponierte Rolle, um einen Migrationshintergrund zu zu schreiben.

Hierbei gilt es zu berücksichtigen, dass die genannten Merkmale nicht allgemein, sondern in spezifischen Ausprägungen als Migrationshinweise gelesen und an Individuen herangetragen werden. So liefern erst die konkreten Merkmalsausprägungen eine Blaupause für die Fremdgruppen- ebenso wie - in ihrer Negation - über die Eigengruppenzuordnung. Für die „Anderen“ stehen beispielsweise Ausprägungen wie dunkle Haut- und Haarfarbe, eine muslimische Religionszugehörigkeit, vermittelt über Kopftuch oder Takke. (Vgl. ebd., S. 98)

Dieses Zeichenreservoir impliziert zugleich die entgegengesetzten Merkmalsausprägungen, welche pauschal der Eigengruppe zugerechnet werden wie helle Hautfarbe oder christliche Religionszugehörigkeit. Die Verfestigung dieser Zuschreibungen und Identifikationsmuster fundiert dementsprechende Homogenitätsvorstellungen über die Eigen- und die Fremdgruppe. In Anlehnung an strukturalistische Ansätze ließe sich auch sagen, dass die benannten Merkmale im Sinne von Zeichen zur Konvention geworden sind und den Gruppenvorstellungen über "uns" und die "anderen « fest zugeordnet bleiben.

Der sich als Interesse am Migrationsanderen (Mecheril, 2010, S. 17) verkleidende, scheinbar unverfänglich-neugierige Fragesatz "Woher kommst Du eigentlich?« kann hierfür als paradigmatisches Kommunikationsbeispiel im Alltag angesehen werden. Einseitig wird eine bestimmte Merkmalsausprägung (Aussehen, Sprachlichkeit, Name) zum Anlass für einen Sprechakt genommen und die implizite Nichtzugehörigkeitszuweisung, der oder die Gefragte komme (im Sinne von: stamme) nicht von "hier" (im Sinne von: Deutschland), wird als Voraussetzung der Frage festgeschrieben. (Vgl. Kunz, 2012, 19 ff.)

Thomas Kunz 
also innerhalb dieses artifiziellen Gruppenkonstrukts, größer sein können als in Bezug auf Personen aus der Gruppe ohne Migrationshintergrund. So können beispielsweise Gemeinsamkeiten zwischen Mitgliedern, die einem gleichen sozialen Milieu zuzurechnen sind, durchaus eine stärkere Bindekraft entwickeln als etwa ein gemeinsamer sogenannter ethnischkultureller Hintergrund (vgl. ebd.).
Ab Mitte der 1950er Jahre initiierte eine staatlich organisierte Anwerbepolitik einen signifikanten Zuzug ausländischer Arbeitskräfte aus Ländern, mit denen die damalige Bundesregierung entsprechende Anwerbeverträge geschlossen hatte: Italien (1955), Spanien und Griechenland (1960), Türkei (1961), Marokko (1963), Portugal (1964), Tunesien (1965) und das frühere Jugoslawien (1968).

\section{"Die neue Zuwanderungsdebatte beherrscht ein nach Nützlichkeits- und Verwertungskriterien differenzierender Duktus"}

Ohnehin erscheint es mit Blick auf das Einbürgerungsgeschehen zunehmend fraglich, ob solche Unterscheidungen noch trennscharf sind. Eine unreflektierte und pauschale Gegenüberstellung der Bevölkerungsgruppen »Deutsche « und »Ausländer«, aber auch »mit « und »ohne Migrationshintergrund « im Sinne eines vermeintlich selbstverständlichen Gegensatzes oder Unterschiedes, der sich aus der Kontextsetzung mit Migration ableitet und die geschilderte Heterogenität und Vielfältigkeit ausblendet, ist demzufolge als problematisch anzusehen und sehr kritisch zu bewerten.

Diese Kritik lässt sich durch die Frage nach der Geltungsdauer des aus heutiger Sicht noch relativ neuen Etiketts Migrationshintergrund noch zuspitzen. Transnationale Migrationsbiographien, hybride Identitäten sowie die, kraft der amtlichen Definition, selbst wieder beschränkte Geltungsdauer des »neuen « Etiketts Migrationshintergrund drängen zu anderen, neuen Bezeichnungspraxen (vgl. Foroutan 2010).

\section{Von der Anwerbe- zur Integrationspolitik}

Die Mitte der 1950er Jahre gilt als markanter Zeitpunkt, der bis heute für die Debatte um Zuwanderung politisch wie demographisch besonders bedeutsam scheint, auch wenn schon unmittelbar nach Ende des Zweiten Weltkrieges mit circa zwölf Millionen deutschen Flüchtlingen und sogenannten Vertriebenen eine nicht geringe Zahl Zuwandernder zu verzeichnen war.
Die Entwicklung der Bundesrepublik hin zu einem sich über die Bedeutung des Zuwanderungsgeschehens bewusst seienden Einwanderungslandes war ein langer Prozess, der sich über die Folgejahrzehnte erstreckte und sich idealtypisch in mehrere Phasen unterteilen lässt. Diese Phasenteilung hat sich in wissenschaftlichen Analysen etabliert, wenn auch mit Unterschieden hinsichtlich der Anzahl der Phasen, den zugeordneten Zeiträumen und inhaltlichen Schwerpunktsetzungen (vgl. Bade/Oltmer, 2004; Meier-Braun, 2006; Butterwegge, 2011).

Jede Phase lässt sich durch einige besonders markante Aspekte charakterisieren. Hierzu zählen, neben den eine jeweilige Phase prägenden Zuwanderungs- und Herkunftsgruppen (vgl. BAMF, 2012), auch spezifische ausländerpolitische Impulse sowie die den Zuwanderungsdiskurs seit je unterlegenden Belastungsoder gar Bedrohungsszenarien (so z. B. in Bezug auf die Gefährdung der Inneren Sicherheit in Deutschland oder das Klischee des Sozialleistungsmissbrauches als Zuwanderungsmotiv).

Auch wenn mittlerweile davon ausgegangen wird, dass die Bundesrepublik Deutschland ein Einwanderungsland ist, sollte dies nicht zu dem Missverständnis führen, $\mathrm{Zu}$ - und Abwanderung als neue Phänomene der letzten 50, 60 Jahre zu begreifen. Vielmehr prägten $\mathrm{Zu}$ - und Abwanderungsprozesse sowie die gesellschaftliche Debatte darum die Gesellschaft in Deutschland auch bereits vor der Gründung der Bundesrepublik.

Die Rezeption tagesaktueller Debatten nährt den falschen Eindruck, Migrations- prozesse als solche fänden bezogen auf Deutschland erst seit Gründung der Bundesrepublik statt. Herbert (2003) bezeichnet dieses Missverständnis als »die Fiktion der Voraussetzungslosigkeit« (Herbert, 2003, S. 9). Untersucht man den Diskurs über Zuwanderung in Deutschland scheine es so, als würde seit den 1970er Jahren in regelmäßigen Abständen und nur leicht variiert die sogenannte Ausländerfrage in den Mittelpunkt der öffentlichen Debatte rücken (vgl. ebd.).

Mögen jene hier nur angedeuteten Variationen auch Hinweise auf thematische Verschiebungen im Zeitverlauf und damit korrespondierend auf den Umgang der sogenannten Mehrheitsgesellschaft mit »ihren« Einwanderern liefern, »tatsächlich [...] wird die Debatte um den Zuzug von Ausländern in Deutschland seit etwa 120 Jahren unter den im wesentlichen gleichen Fragestellungen und mit den gleichen Frontlinien geführt." (Ebd.; vgl. auch Bade/Oltmer, 2004, S. 5 ff.)

\section{Vom späten Eingeständnis, Einwanderungsland zu sein}

Das Jahr 1998 markierte einen Wendepunkt in der Debatte um das Selbstverständnis, ein Einwanderungsland zu sein. Mit der damaligen Wahl einer rot-grünen Bundesregierung wurde das Bekenntnis, ein Einwanderungsland zu sein, programmatische Grundlage der Ausländerpolitik auf Bundesebene. Zugleich zeichnete sich ab, dass Ausländerpolitik in der Einwanderungsgesellschaft integrationspolitischen Aspekten auf Bundesebene zukünftig ausdrücklich stärkere Bedeutung beimessen würde.

All dies materialisierte sich in Gestalt der Erarbeitung, der parlamentarischen Verhandlung und schließlich dem Inkrafttreten des sogenannten Zuwanderungsgesetzes im Jahr 2005 - dem eine heftige innenpolitische Kontroverse sowie ein verfassungsrechtlicher Streit voraus gingen. Besonderheit dieses Gesetzes war neben der Bündelung verschiedener ausländerrechtlicher Regelungen in einem einzigen Gesetz der exponierte Einbezug integrationspolitischer Maßnahmen. (Vgl. Meier-Braun, 2006, S. 206 f.; Butterwegge, 2011, S. 25 f.).

Gleichzeitig wurden die Diskussionen um Details und Ausgestaltung jenes neuen Gesetzes auch durch »9/11", also die Anschläge auf das World Trade Center in New York, beeinflusst. Ein Ereignis, 
welches ebenso eine Zäsur markierte, allerdings im Sinne eines Rückschrittes, da fortan die Frage der sogenannten Terrorbekämpfung den Migrationsdiskurs zunehmend mitbestimmte. "9/11 « bedeutete somit eine verstärkte inhaltliche Verschiebung hin zu Sicherheitsfragen sowie eine Intensivierung teils bereits bekannter Bedrohungsrhetoriken, die sich insbesondere durch eine Kontextsetzung mit islamischer Religionszugehörigkeit auszeichneten. Beides wirkt(e) sich auf das Zuwanderungsgesetz im speziellen und die gesellschaftliche Diskussion des Migrationsthemas im Allgemeinen aus.

\section{Deutschland - widerwilliges Einwanderungsland}

Selbst wenn sich die Bundesrepublik nun auch auf politischer Ebene und parteienübergreifend Einwanderungsland nennt, ist diese Selbstbezeichnung angesichts anhaltender Einwände und seitens Einwanderungsskeptikern und Einwanderungsgegnern propagierten Bedrohungsszenarien ein Euphemismus. Es ist ein Euphemismus, soweit damit nahelegt wird, die Bundesrepublik sei ein Land, welches $\mathrm{Zu}$ - und Einwanderung leicht mache oder gar befördere. Vielmehr ist das Gegenteil der Fall: Begrenzung, Selektion, Abwehr, Erschwerung und Illegalisierung sind Stichworte, welche viel eher das herrschende Zuwanderungsregime charakterisieren.

So betrachtet erscheint die Bezeichnung »widerwilliges Einwanderungsland « (Bade, 2007, S. 33) angemessener. Dieser Widerspruch wird auch an der Bezeichnung des nach einigem innenpolitischen Hin und Her in Kraft getretenen Gesetzes deutlich. Zwar wird es im öffentlichen Sprachgebrauch "Zuwanderungsgesetz « genannt, der tatsächliche und vollständige Titel lautet indes: »Gesetz zur Steuerung und Begrenzung der Zuwanderung und zur Regelung des Aufenthalts und der Integration von Unionsbürgern und Ausländern «.

Aber auch die Quasi-Verpolizeilichung der Flüchtlings- und Asylpolitik der Europäischen Union in Gestalt der Einrichtung und Arbeit der Europäische Agentur für die operative Zusammenarbeit an den Außengrenzen, bekannt unter dem Akronym FRONTEX (abgeleitet von »Frontières extérieures «, dem französischen Wort für Außengrenzen), ist ein Beispiel für diese Dynamik, deren abweh- render Charakter sich mit dem Stichwort »Festung Europa « holzschnittartig, aber treffend beschreiben lässt.

Zunehmend wird auch EU-Binnenmigration als bedrohlich gerahmt, nachdem die Eurokrise und die daraus resultierenden gesellschaftlichen Konflikte in den betroffenen Mitgliedstaaten zu einer Zunahme des Wanderungsgeschehens geführt haben. Der Begriff Festung ist jedoch insofern irreführend, als damit keine pauschale Abschottung gegen $\mathrm{Zu}$ wanderung gemeint ist, als vielmehr ein nach Nützlichkeits- und Verwertungskriterien differenzierendes, durchaus flexibel und dynamisch agierendes $\mathrm{Zu}$ wanderungsregime. Auf insbesondere ökonomisch indizierte Arbeitskräftebedarfe in den Mitgliedsstaaten wird mit selektiver Durchlässigkeit reagiert, Zuwanderungsversuche von als nicht wünschenswert identifizierten Migrantinnen und Migranten werden an den EU-Außengrenzen mit umfassenden paramilitärischen Mitteln bekämpft.

\section{Vom Eingeständnis zum angemessenen Selbstverständnis eines Einwanderungslandes}

Nachdem das widerwillige Eingeständnis, ein Einwanderungsland zu sein, gewissermaßen Staatsdoktrin wurde, wird die gegenwärtige Phase geprägt durch den gesellschaftlichen Anpassungs- und Umstellungsprozess, den dieses Eingeständ- neuen 'Wir«" (Hirseland/Chlihi, 2013, 25) befindet, eine zunehmende Rolle. Diese Diskussion schlägt sich in neuen Bezeichnungsversuchen nieder, wie beispielsweise der Formulierung von den »neuen Deutschen « (Bota et al., 2012) oder sie wird in staatstragenden Reden aufgegriffen, wie die Ausführungen von Bundespräsident Gauck im Mai diesen Jahres belegten, der bei einer Einbürgerungsfeier anlässlich 65 Jahre Grundgesetz u. a. "ein neues deutsches /Wir« « (Gauck, 2014, 6) konstatierte.

Doch auch anhaltende Ab- und Ausgrenzungsversuche seitens einer sich immer noch als Mehrheitsgesellschaft konstruierenden Bevölkerung gegenüber staatsbürgerrechtlich längst $\mathrm{Da}$ zugehörigen durchziehen und prägen die gegenwärtige Phase. Das zu einer gewissen Prominenz gelangte Menetekel »Deutschland schafft sich ab« und die in dessen Folge aufgekommene Diskussion kann in diesem Kontext als hilflos-aggressives Beharren auf nationalen Homogenitätsvorstellungen gelesen werden, die sich dem Erfordernis einer Revision des bisherigen nationalen Selbstverständnisses widersetzen, das einer Einwanderungsgesellschaft bereits seit längerem nicht angemessen ist.

Eingelassen in die gesellschaftliche Auseinandersetzung um diese Neubestimmung sind dramatisierende Warnungen vor den Folgen bisheriger und zukünftiger Zuwanderung. Diese Bedrohungsszenari-

\section{"Die Integrationsdebatte durchzieht ein} Gegenüber von swir und sihnen"

nis erforderlich macht. Nicht zuletzt der sich immer mehr bemerkbar machende Umstand der Veränderung der demographischen Zusammensetzung (Stichwort: Migrationshintergrund) erfordert ein Umdenken hinsichtlich bislang zugrunde gelegter Vorstellungen von Mehrheitsoder Aufnahmegesellschaft, eine Revision paternalistisch-einseitiger Anpassungserwartungen sowie eine Umstellung auf adäquate Anerkennungskulturen und Anerkennungspolitiken - und entsprechende Begrifflichkeiten (vgl. hierzu beispielsweise Foroutan, 2010).

In unterschiedlichen Kontexten spielt die Frage, ob »sich Deutschland aufgrund seiner Vielfalt auf dem Weg zu einem en kreisen auffällig um die Sicherheitslage und deren Gefährdung durch Migrationsgeschehen und Migrantinnen und Migranten im Innen wie im Außen.

\section{Deutschland schafft sich $a b$ ? Na endlich!}

Die Frage »Wer sind wir? « variiert ein Kernthema abseits geläufiger Überlegungen über Zu-, Abwanderungs- und Aufnahmezahlen, über sogenannte Integrationsmaßnahmen und deren Wahrnehmung. Es steht aufgrund der historischen Dauer der »neuen Zuwanderung «, also der Zuwanderung in den vergangenen fast 60 Jahren die Beantwortung der 
Frage an, ab wann Menschen, die seit 50 Jahren in Deutschland leben, die hier geboren und aufgewachsen sind, faktisch dazugehören (können) und unhinterfragt zugehörig sind.

Unterschieden wird immer noch und scheinbar selbstverständlich zwischen einem imaginierten "wir« und "uns « (als den Angehörigen der sogenannten Mehrheitsgesellschaft) sowie »ihnen « (als den Angehörigen der Gruppe der Zugewanderten, der Migrantinnen und Migranten). Diese Zuweisung ist implizit national konnotiert. »Wir«, damit sind in der Regel Deutsche ohne Migrationshintergrund gemeint. Diese Kollektivgruppeninszenierung unterläuft allerdings selbst die mittels Einbürgerung vollzogene staatsbürgerrechtliche Gleichstellung von Personen, die über eine Migrationsgeschichte verfügen. Diese bleiben weiterhin unterschieden quasi als Deutsche zweiter Klasse. Mag man den Pass auch besitzen, man gehört dennoch nicht zu "uns", sondern zu »ihnen«.

Wichtig scheint deshalb eine Verständigung und Vergewisserung darüber, wie die mittels der Integrationsdebatte erzeugte und permanent reproduzierte diskursive Frontstellung zwischen »uns« (Menschen ohne Migrationshintergrund) und »ihnen« (Menschen mit Migrationshintergrund) zu bestreiten, durcheinander zu bringen und nachhaltig zu irritieren ist. Das erscheint meines Erachtens als das zentrale Erfordernis, um den diskursiven Raum zu öffnen, um die umrissenen semantischen Frontstellungen und Binaritäten aufzubrechen. Es wäre die Grundlage für ein neues und einer Einwanderungsgesellschaft angemesseneres »wir" - wohlgemerkt: "wir" immer in Anführungszeichen.

Damit ist die Aktualisierung der Antwort auf die Frage gemeint, wer sich eigentlich zu dem ominösen »wir « rechnen darf und wer demgegenüber als nichtzugehöriges »sie « konstruiert wird. Denn egal was Menschen, die mittels des Etiketts »Migrationshintergrund « öffentlich und auf Dauer als Andere kommuniziert werden, auch tun oder noch tun werden, sie werden gegenwärtig lediglich als entweder "gut « oder »schlecht" integrierte, aber in beiden Fällen als national eigentlich Nichtzugehörige positioniert.

In diesem Kontext kann rhetorisch die im Zuge der Sarrazin-Debatte zu fragwürdiger Berühmtheit geratene Behauptung »Deutschland schafft sich $\mathrm{ab}$ « nochmals aufgegriffen werden. Diese lässt sich gegenläufig zur Absicht ihrer Verfechter hinsichtlich ihres provokanten Gehaltes abklopfen, um nämlich umgekehrt zu überlegen, inwieweit diese Feststellung in einem kritischen Sinne nicht durchaus positiv zu deuten ist.

Es gilt hierbei auch in den Blick zu nehmen, wie die Wahrnehmung von »ihnen« als bevölkerungspolitisch identifizierbarer Sondergruppe durch eine vorherrschend einseitig auf Migranten und deren tatsächliches oder angebliches Integrationsverhalten ausgerichtete Beobachtung (und Gültigmachung von individualisierter Verantwortungszuweisung) etabliert wird. Die als solche immer weniger eindeutig bestimmbare, weil selbst von Vielfalt und Zuwanderungsgeschichten und Zuwanderungsbiographien geprägte und durchzogene sogenannte Mehrheitsgesellschaft versucht sich mittels solcher Positionszuweisungen, also der Produktion der »Anderen «, implizit ihrer prekär gewordenen und faktisch verblassenden ethnisch-nationalen Homogenitätsphantasien zu versichern, diese gleichsam trotzig zu beschwören. So betrachtet leistet der herrschende Blick auf die Migrantinnen und Migranten (oder die Muslime - was häufig synonym verwendet wird) und deren Integrationsverhalten vor allem eine Selbstvergewisserung und Kohäsionsleistung der sogenannten Mehrheitsgesellschaft im Sinne der Staatsbürgergemeinschaft.

Die Feststellung, die Sarrazin seinerzeit nutzte, um sich und seine ressentimentgeladenen Thesen geschickt im öffentlichen Diskurs zu lancieren, reklamierte für sich die Definitionshoheit darüber, was denn dieses Deutschland sei. Eben ein Staat oder eine Gesellschaft, welche in den Augen des Verfechters dieser These - und derer, die sie bereitwillig aufgriffen - gleichzusetzen sei mit einer Bevölkerung, die sich just durch jene, demografisch längst schon obsolet gewordene nationale Homogenitätsfiktion charakterisieren ließe. Sarrazin und Co. versuchten letztlich nichts anderes, als ein bevölkerungspolitisches Reinheitsphantasma zu beschwören oder zu restaurieren, welches de facto immer schon ein Konstrukt war.

Es mag paradox anmuten, vor diesem Hintergrund kann das in der Öffentlichkeit zu beobachtende Auftauchen jener These, nun aus kritischer Perspektive, positiv gedeutet werden: Weil die Fest- stellung, Deutschland schaffe sich ab, als starkes Indiz für den - zumindest von rechtskonservativer Seite - zu beklagenden Verlust oder für die gegenwärtige Schwächung einer völkisch-nationalen Ideologie als notwendigem Kernbestand kollektiver Identitätsbildung interpretiert werden kann. Es ist dies ein Verlust oder eine Schwächung, die es für die Verfechter jener Ideologie nötig erscheinen lässt, den Verlust eben dieser zu skandalisieren.

Wenn Akteure diese Entwicklung als bedrohlich zu vermitteln versuchen wie just durch Sarrazin geschehen -, ist diesen, in Antwort auf ihr Menetekel »Deutschland schafft sich $a b$ «, entgegen zu halten: »Na endlich!«

Abschaffungswürdig und zu begrüBen nämlich insofern, als es sich dabei um den längst überfälligen Abschied von einer ressentimentgeladenen und völkischen Sichtweise auf die bundesrepublikanische Wohnbevölkerung handelt. Eine Sichtweise und Haltung, die sich unter anderem dadurch auszeichnete, sich über lange Zeit der Einsicht verweigert zu haben, dass nicht eine hier lebende Spezialbevölkerungsgruppe einen »Migrationshintergrund « oder was auch immer hat, sondern die sogenannte Mehrheitsgesellschaft selber.

\section{Literatur}

Bade, Klaus J./Oltmer, Jochen (2004): Normalfall Migration, Bonn

Bade, Klaus J. (2007): Versäumte Integrationschancen und nachholende Integrationspolitik. In: APuZ - Aus Politik und Zeitgeschichte,

Nr. 22-23/2007, S. 32-38.

BAMF - Bundesamt für Migration und Flüchtlinge (Hg.) (2009): Working Paper 27 der Forschungsgruppe des Bundesamtes. Grunddaten der Zuwandererbevölkerung in Deutschland, Nürnberg.

BAMF - Bundesamt für Migration und Flüchtlinge (Hg.) (2012): Das Bundesamt in Zahlen 2011. Asyl, Migration, ausländische Bevölkerung und Integration, Nürnberg.

\section{BMI - Bundesministerium des Innern ( $\mathrm{Hg}$.)}

(2014): Migrationsbericht des Bundesamtes für Migration und Flüchtlinge im Auftrag der Bundesregierung. Migrationsbericht 2012, Nürnberg. Bota, Alice/Pham, Khuê/Topçu, Özlem (2012): Wir neuen Deutschen. Wer wir sind, was wir wollen, Reinbeck.

Butterwegge, Carolin (2011): Zuwanderung in Deutschland. Eine historische Betrachtung des Wanderungsgeschehens und der Migrations- 
politik. In: Thomas Kunz, Ria Puhl (Hg.): Arbeitsfeld Interkulturalität. Grundlagen, Methoden und Praxisansätze der Sozialen Arbeit in der Zuwanderungsgesellschaft, S. 16-31, Weinheim. Foroutan, Naika (2010): Neue Deutsche, Postmigranten und Bindungs-Identitäten. In: APuZ - Aus Politik und Zeitgeschichte, Nr. 46-47/2010, S. 9-15.

Gauck, Joachim (2014): Rede bei der Einbürgerungsfeier anlässlich 65 Jahre Grundgesetz am 22. Mai 2014 im Schloss Belevue. www.bundespraesident.de/ SharedDocs/Downloads/DE/Reden/2014/05/ 140522-Einbuergerung-Integration.pdf? blob=publicationFile (Download 24.06.2014). Herbert, Ulrich (2003): Geschichte der Ausländerpolitik in Deutschland. Schriftenreihe. Band 410, Bonn.

Hirseland, Katrin/Chlihi, Zakia (2013): "Ja, wir verändern uns - und das ist auch gut so! « Die Nürnberger Tage für Integration füllen den Begriff "Anerkennungskultur " mit Leben“. In: Blickpunkt Integration 2013, S. 24-25.

Kunz, Thomas (2008): "Kartoffelgrafik jetzt auch mit Migrationshintergrund. Die Über- tragung des Sinus-Milieu-Ansatzes auf die Bevölkerung mit Migrationshintergrund in Deutschland «. In: Migration und Soziale Arbeit, Heft 1/2008, S. 69-72.

Kunz, Thomas (2011): Gschichten von "uns" und »ihnen«. Die Reflexion gesellschaftlicher Fremdheitsbilder als Bestandteil Interkultureller Kompetenz. In: Thomas Kunz, Ria Puhl (Hg.): Arbeitsfeld Interkulturalität. Grundlagen, Methoden und Praxisansätze der Sozialen Arbeit in der Zuwanderungsgesellschaft, S. 90-106, Weinheim.

Kunz, Thomas (2012): "Woher kommst Du eigentlich?" Das Interesse an Herkunft als Zuweisung von Zugehörigkeit und Nichtzugehörigkeit. In: Sozialmagazin, 4/2012, S. 19-27. Meier-Braun, Karl-Heinz (2006): Der lange Weg ins Einwanderungsland Deutschland. In: Zuwanderung und Integration, Der Bürger im Staat, Heft 4/2006, S. 204-209.

Mecheril, Paul (2010): Migrationspädagogik. Hinführung zu einer Perspektive. In: Paul Mecheril, María do Mar Castro Varela, İnci Dirim, Annita Kalpaka, Claus Melter: Migrationspädagogik, S. 7-22, Weinheim und Basel.
Schröer, Wolfgang/Schweppe, Cornelia (2010): Transmigration und Soziale Arbeit - ein öffnender Blick auf die Alltagswelten im Kontext von Migration. In: Migration und Soziale Arbeit, Heft 2/2010, S. 91-97.

Statistisches Bundesamt (Hg.) (2012): Bevölkerung und Erwerbstätigkeit. Bevölkerung mit Migrationshintergrund - Ergebnisse des Mikrozensus 2011 - Fachserie 1, Reihe 2.2. Wiesbaden.

\section{Statistisches Bundesamt (Hg.) (2013):}

Zensus 2011 - Bevölkerung Bundesrepublik Deutschland am 9. Mai 2011, Wiesbaden (www.destatis.de/DE/PresseService/Presse/ Pressekonferenzen/2013/Zensus2011/ bevoelkerung_zensus2011.pdf;jsessionid=B9C 9429BBoDCAFD3Eo61BEE663FB6oo3.cae3? blob=publicationFile (Download 31.05.2013). Statistisches Bundesamt (Hg.) (2014): Zensus 2011 - Bevölkerung \& Haushalte. Übersicht über Merkmale und Merkmalsausprägungen, Definitionen. Stand: 28.05.2014 (www. zensus2011.de/SharedDocs/Downloads/ DE/Merkmale/Merkmale_Bevoelkerung pdf? _blob=publicationFile \&v=9 (Download 19.07.2014)

\section{Neuausgabe 2014}

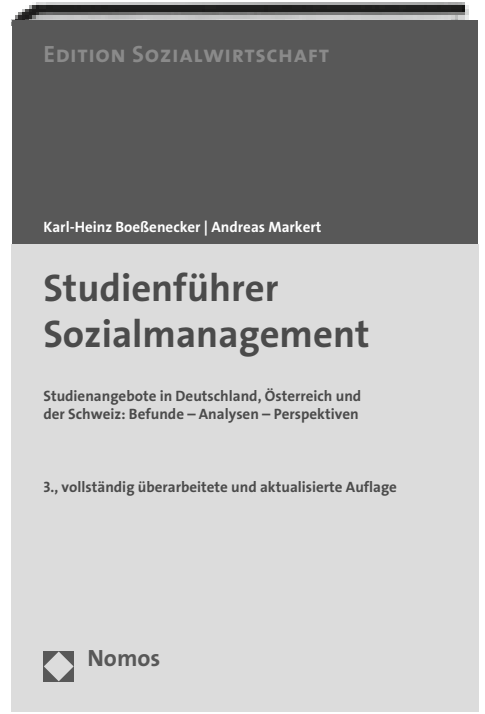

\author{
Studienführer Sozialmanagement \\ Studienangebote in Deutschland, Österreich und der Schweiz: \\ Befunde - Analysen - Perspektiven \\ Von Prof. Dr. Karl-Heinz Boeßenecker und Prof. Dr. Andreas Markert \\ 3., vollständig überarbeitete und aktualisierte Auflage 2014, \\ 221 S., brosch., 24,- $-€$ \\ ISBN 978-3-8487-0956-4 \\ (Edition Sozialwirtschaft, Bd. 19) \\ www.nomos-shop.de/21806
}

Die Autoren rekonstruieren in der vollständig überarbeiteten und aktualisierten 3. Auflage des Studienführers die an Hochschulen Deutschlands, der Schweiz und Österreichs bestehenden Studiengänge im Bereich Sozialmanagement/Sozialwirtschaft und ordnen diese fachlich ein. Studierende, Lehrende und Praktiker/Praktikerinnen erhalten so eine systematische Übersicht über ein expandierendes Studienund Handlungsfeld.

Bestellen Sie jetzt telefonisch unter 07221/2104-37.

Portofreie Buch-Bestellungen unter www.nomos-shop.de 